\title{
A Unique Analytical Solution of the White Matter Standard Model using Linear and Planar Encodings
}

\author{
Marco Reisert ${ }^{1,2,3}$, Valerij G. Kiselev ${ }^{1,3}$, and Bibek Dhital ${ }^{1,3,4}$ \\ ${ }^{1}$ Medical Center, Faculty of Medicine, University Freiburg, Germany \\ ${ }^{2}$ Department of Functional and Stereotactic Neurosurgery, Freiburg \\ ${ }^{3}$ Department of Medical Physics, Freiburg \\ ${ }^{4}$ Department of Neurophysics, Max Planck Institute for Human Cognition and \\ Brain Sciences, Leipzig, Germany
}

September 20, 2018

\begin{abstract}
Diffusion-weighted magnetic resonance imaging in brain white matter probes tissue microstructure and allows for the estimation of compartmental diffusion parameters. Recently, it became apparent that traditional single-direction diffusion encodings are not fully sufficient to resolve the white matter compartmental diffusivities. Multiple diffusion encodings have been suggested to make the problem less ambiguous, however, it still remained unclear whether such protocols would completely solve the problem. Here, we constructively prove that a combination of linear and planar diffusion encodings is enough to determine the parameters of the three compartment white matter model.
\end{abstract}

\section{Introduction}

For a long time, attempts to multi-compartment modeling in brain white matter (WM) with simple single diffusion encodings [Fieremans et al., 2011, Zhang et al., 2012, Novikov et al., 2018, Reisert et al., 2017] led to ambiguous results [Jelescu et al., 2016, Novikov et al., 2018]. For example, it was argued in [Fieremans et al., 2011] that intra axonal diffusion is substantially smaller than extra axonal diffusion along the axons, while others argued for the opposite [Zhang et al., 2012, Dhital et al., 2017]. Multiple diffusion encodings offer substantially more information than ordinary single diffusion encoding schemes [Jespersen et al., 2013, Westin et al., 2014]. However, most efforts in understanding the additional information gained by such methods were focused on dispersed single-compartment systems thus revealing apparent measures like eccentricity, microscopic and fractional anisotropy [Jespersen et al., 2013, Westin et al., 2014, Szczepankiewicz et al., 2015].

Recent studies have investigated the benefits of using multiple diffusions encodings to resolve white matter compartmental parameters [Lampinen et al., 2017, Dhital et al., 2018]. For example, spherical diffusion encodings [Dhital et al., 2017] shows very low kurtosis in white and gray matter, which gives rise to the assumption that traces of the tissue compartments are similar. In [Fieremans et al., 2018] an additional spherical encodings were used to stabilize fits and release constraints. Or, in [Coelho et al., 2017, Reisert et al., 2018] a combination of linear and planar encodings was used with the same intention. Thus, the question arises, what kind of protocol is sufficient to solve the problem uniquely? This short note contributes to the answer of this question.

We will show that a combination of linear and planar encodings is indeed enough to provide a unique solution of the full 3-compartment model of brain white matter using $\mathcal{O}\left(b^{2}\right)$ measurements. The key ingredient of the approach is that a combination of linear and planar measurements provide a direct estimate of the mesoscopic orientation dispersion, without relying on any other concurrent estimates. We further discuss an inherent model property, that, under special conditions, this solution still shows an ambiguity. Finally, we demonstrate by a few counterexamples the inadequacy of linear and spherical encoding to resolve the problem ( taking only $\mathcal{O}\left(b^{2}\right)$ coefficients). 


\section{The White Matter Model}

We follow the standard tissue model as proposed in [Novikov et al., 2018, Reisert et al., 2017]. In contrast to [Zhang et al., 2012], in this model both intra and extra-axonal compartments undergo the same convolution with the mesoscopic orientation distribution. For a general encoding matrix B the signal for this model looks as follows

$$
\begin{aligned}
S(\mathbf{B}) & =\int_{\mathbf{n} \in S_{2}} d^{2} \mathbf{n} M(\mathbf{n}, \mathbf{B}) f(\mathbf{n}) \\
& =\int_{\mathbf{n} \in S_{2}} d^{2} \mathbf{n}\left(v_{i} e^{-\operatorname{tr}\left(\mathbf{B D}_{i}^{\mathbf{n}}\right)}+v_{e} e^{-\operatorname{tr}\left(\mathbf{B D}_{e}^{\mathbf{n}}\right)}+v_{\mathrm{f}} e^{-\operatorname{tr}(\mathbf{B}) D_{\mathrm{f}}}\right) f(\mathbf{n})
\end{aligned}
$$

where $f(\mathbf{n})$ is an arbitrary, normalized orientation distribution function and $M(\mathbf{n}, \mathbf{B})$ the axially symmetric, multi-exponential microstructural model with symmetry axis $\mathbf{n}$. The diffusion tensor of intra- and extra-axonal fractions are parametrized as

$$
\mathbf{D}_{i}^{\mathbf{n}}=\mathbf{n} \mathbf{n}^{T} D_{i}, \mathbf{D}_{e}^{\mathbf{n}}=\mathbf{n n}^{T} \Delta_{e}+\mathbf{I}_{3} D_{e}
$$

We now focus on linear encoding $\mathbf{B}_{\text {lin }}=b \mathbf{q} \mathbf{q}^{T}$ and planar encoding $\mathbf{B}_{\text {pla }}=b\left(\mathbf{I}_{3}-\mathbf{q} \mathbf{q}^{T}\right) / 2$, where $\mathbf{q}$ is the diffusion gradient direction of modulus one and the b-value $b$ is defined as the trace of the b-matrix. Rewriting the microstructural model in terms of the cosine $t=\mathbf{q}^{T} \mathbf{n}$ between encoding direction and axon orientation gives,

$$
\begin{aligned}
& M_{\text {lin }}(t, b)=v_{\mathrm{i}} e^{-b D_{i} t^{2}}+v_{\mathrm{e}} e^{-b \Delta_{e} t^{2}-b D_{e}}+v_{\mathrm{f}} e^{-b D_{\mathrm{f}}} \\
& M_{\text {pla }}(t, b)=v_{\mathrm{i}} e^{-b D_{i}\left(1-t^{2}\right) / 2}+v_{\mathrm{e}} e^{-b \Delta_{e}\left(1-t^{2}\right) / 2-b D_{e}}+v_{\mathrm{f}} e^{-b D_{\mathrm{f}}}
\end{aligned}
$$

In this formulation, the convolution with the mesostructural orientation distribution $f(\mathbf{n})$ takes the form

$$
S_{\alpha}(\mathbf{q}, b)=\int_{\mathbf{n} \in S_{2}} d^{2} \mathbf{n} f(\mathbf{n}) M_{\alpha}\left(\mathbf{q}^{T} \mathbf{n}, b\right)
$$

where $\alpha=$ linear or $\alpha=$ planar depending on the gradient waveform. Note that $S_{\alpha}(\mathbf{q}, b)$ is normalized in the sense $S_{\alpha}(\mathbf{q}, 0)=1$.

The key to decouple micro and mesostructural contribution is to work in the domain of spherical harmonics. The spherical convolution turns out to be a product of the two spherical harmonic representations, $f_{l, m}$ and $M_{\alpha}^{l}(b)$, of $f(\mathbf{n})$ and $M_{\alpha}(t, b)$, respectively.

$$
S_{\alpha}^{l, m}(b)=f_{l, m} M_{\alpha}^{l}(b) .
$$

We used here in semi-Schmidt normalization ${ }^{1}$ as in [Reisert et al., 2017]. The signal is characterized by a set of quantities that are rotationally invariant for any signal-generating tissue.

$$
S_{\alpha}^{l}(b)=\sqrt{\sum_{m=-l}^{l}\left|S_{\alpha}^{l, m}(b)\right|^{2}}=f_{l}\left|M_{\alpha}^{l}(b)\right|
$$

Here $f_{l}=\sqrt{\sum_{m}\left|f_{l, m}\right|^{2}}>0$ is the rotation invariant mesoscopic dispersion. For both linear and planar encodings, we define the moments

$$
\begin{aligned}
W_{\alpha}^{l, k} & :=\left.\frac{1}{4 \pi} \frac{d^{k}}{d b^{k}}\right|_{b=0} S_{\alpha}^{l}(b) \\
& =\left.f_{l} \operatorname{sgn}\left(M_{\alpha}^{l}(0)\right) \int_{-1}^{1} \frac{d t}{2} P_{l}(t) \frac{d^{k}}{d b^{k}}\right|_{b=0} M_{\alpha}(t, b),
\end{aligned}
$$

\footnotetext{
1 In this normalization $\sum_{m=-l}^{l}\left|Y_{l}^{m}(\mathbf{n})\right|^{2}=1$ and $\int d^{2} \mathbf{n} Y_{l}^{m}(\mathbf{n}) Y_{l^{\prime}}^{m^{\prime}}(\mathbf{n})^{*}=\frac{4 \pi}{2 l+1} \delta_{l, l^{\prime}} \delta_{m, m^{\prime}}$ and $Y_{l}^{0}(\mathbf{n})=$ $P_{l}(\cos \theta)$, where $Y_{l}^{m}$ are the spherical harmonics and $P_{l}$ the Legendre polynomials and $\theta$ the polar angle of $\mathbf{n}$. The axial symmetry implies that the spherical harmonics expansion of $M_{\alpha}\left(\mathbf{q}^{T} \mathbf{n}, b\right)$ contains only components with $l=0, M_{\alpha}\left(\mathbf{q}^{T} \mathbf{n}, b\right)=\sum_{l} \frac{2 l+1}{4 \pi} M_{\alpha}^{l}(b) P_{l}\left(\mathbf{q}^{T} \mathbf{n}\right)$.
} 
where $\operatorname{sgn}(x)=x /|x|$ and we do not write the delta-functional term for $l \geq 2$, since $M_{\alpha}^{l}(b)$ have definite signs. This follows from their physical meaning of the signal from the idealized unidirectional fiber bundle, since diffusion is faster along such a bundle, $M_{\text {lin }}^{2}<0$ and $M_{\text {pla }}^{2}>0$, for all meaningful constellations of microstructural parameters. Introduction of these definite signs is sufficient to resolve the ambiguity borne by taking the square of Eq. (7), which is necessary to build rotation invariant quantities.

Note that the moments defined in Eq. (8) generalize the moments used by [Novikov et al., 2018]; for linear encoding $W_{\text {lin }}^{l, k} \propto M^{(2 k), l}$ following definitions in [Novikov et al., 2018] equations (15-18).

\subsection{Finding the solution}

The white matter model described above includes one known (the free water diffusivity, $D_{\mathrm{f}}$ ) and five unknown scalar parameters: intra-axonal difusivity $\left(D_{i}\right)$, extra-axonal radial diffusivity $\left(D_{e}\right)$, difference between extra-axonal parallel and radial diffusivity $\left(\Delta_{e}\right)$, and the volume fractions $\left(v_{\mathrm{i}}\right.$, $\left.v_{\mathrm{e}}, v_{\mathrm{f}}\right)$ with the constraint $v_{i}+v_{e}+v_{f}=1$. The orientation distribution function $f(\mathbf{n})$ contains an infinite set of coefficients. In this section we show that resolving the signal for both linear and planar encoding up to the order $b^{2}$ and $l=2$ enables unambiguous determination of the scalar parameters and the first non-trivial coefficient, $f_{2}$, of $f(\mathbf{n})$.

The corresponding moments are expressed via the model parameters as follows:

$$
\begin{aligned}
W_{\mathrm{lin}}^{0,1} & =-\frac{1}{3} \Delta_{e} v_{\mathrm{e}}-D_{e} v_{\mathrm{e}}-\frac{1}{3} D_{i} v_{\mathrm{i}}-D_{\mathrm{f}} v_{\mathrm{f}} \\
W_{\mathrm{lin}}^{2,1} & =\frac{2}{15} f_{2}\left[\Delta_{e} v_{\mathrm{e}}+D_{i} v_{\mathrm{i}}\right] \\
W_{\mathrm{lin}}^{0,2} & =\frac{1}{5} \Delta_{e}^{2} v_{\mathrm{e}}+D_{e}^{2} v_{\mathrm{e}}+\frac{1}{5} D_{i}^{2} v_{\mathrm{i}}+D_{\mathrm{f}}^{2} v_{\mathrm{f}}+\frac{2}{3} \Delta_{e} D_{e} v_{\mathrm{e}} \\
W_{\mathrm{lin}}^{2,2} & =-f_{2}\left[\frac{4}{35} \Delta_{e}^{2} v_{\mathrm{e}}+\frac{4}{35} D_{i}^{2} v_{\mathrm{i}}+\frac{4}{15} \Delta_{e} D_{e} v_{\mathrm{e}}\right] \\
W_{\mathrm{pla}}^{0,2} & =\frac{2}{15} \Delta_{e}^{2} v_{\mathrm{e}}+D_{e}^{2} v_{\mathrm{e}}+\frac{2}{15} D_{i}^{2} v_{\mathrm{i}}+D_{\mathrm{f}}^{2} v_{\mathrm{f}}+\frac{2}{3} \Delta_{e} D_{e} v_{\mathrm{e}} \\
W_{\mathrm{pla}}^{2,2} & =-f_{2}\left[\frac{4}{105} \Delta_{e}^{2} v_{\mathrm{e}}+\frac{4}{105} D_{i}^{2} v_{\mathrm{i}}+\frac{2}{15} \Delta_{e} D_{e} v_{\mathrm{e}}\right]
\end{aligned}
$$

The calculations straightforwardly follow from, Eq. (8). Note the absence of the moments $W_{\text {pla }}^{l, 1}$ in this order (linear in $b$ ) measurements with any shape of $\mathbf{B}$ is equivalent to a set of single-direction measurements and thus do not add any extra information. For example, the signal obtained using the planar encoding in the $x, y$ plane is equivalent to the mean of signals encoded linearly in the $x$ and $y$ directions. In particular, $W_{\text {lin }}^{0,1}=W_{\text {pla }}^{0,1}$ and $W_{\text {lin }}^{2,1}=2 W_{\text {pla }}^{2,1}$, which can be observed from the fact that $M_{\text {pla }}(t, b)$, Eq. (4), can be obtained from $M_{\text {lin }}(t, b)$, Eq. (3), by substituting $t^{2}$ with $\left(1-t^{2}\right) / 2=\left[P_{0}(t)-P_{2}(t)\right] / 3$. Complimentary information can be found in the second (or higher) order of $b$.

The dispersion parameter, $f_{2}$, can be easily found from Eq. (12)-Eq. (15)

$$
f_{2}=-\frac{7}{4} \frac{W_{\mathrm{lin}}^{2,2}-2 W_{\mathrm{pla}}^{2,2}}{W_{\mathrm{lin}}^{0,2}-W_{\mathrm{pla}}^{0,2}}
$$

Note that the denominator is just the average eccentricity of the compartments [Jespersen et al., 2013] (or microstructural fractional anisotropy), namely $W_{\text {lin }}^{0,2}-W_{\text {pla }}^{0,2}=\Delta_{e}^{2} v_{\mathrm{e}}+D_{i}^{2} v_{\mathrm{i}}$.

Finding other parameters is not so straightforward. Assuming $f_{2}$ is known, we define a set of 
auxiliary variables $x_{i}$ as follows

$$
\begin{array}{ll}
x_{1}=\frac{15}{2 f_{2}} W_{\text {lin }}^{2,1} & =\Delta_{e} v_{\mathrm{e}}+D_{i} v_{\mathrm{i}} \\
x_{2}=W_{\text {lin }}^{0,2}-W_{\mathrm{pla}}^{0,2} & =\Delta_{e}^{2} v_{\mathrm{e}}+D_{i}^{2} v_{\mathrm{i}} \\
x_{3}=-W_{\mathrm{lin}}^{0,1}-\frac{5}{2 f_{2}} W_{\text {lin }}^{2,1} & =D_{e} v_{\mathrm{e}}+D_{\mathrm{f}} v_{\mathrm{f}} \\
x_{4}=W_{\text {lin }}^{0,2}+\frac{1}{4 f_{2}} W_{\mathrm{lin}}^{2,2}+\frac{9}{2 f_{2}} W_{\mathrm{pla}}^{2,2} & =D_{e}^{2} v_{\mathrm{e}}+D_{\mathrm{f}}^{2} v_{\mathrm{f}} \\
x_{5}=\frac{15}{2 f_{2}} W_{\text {lin }}^{2,2}-\frac{45}{2 f_{2}} W_{\mathrm{pla}}^{2,2} & =\Delta_{e} D_{e} v_{\mathrm{e}}
\end{array}
$$

This system including the constraint on the compartment water fractions

$$
v_{\mathrm{i}}+v_{\mathrm{e}}+v_{\mathrm{f}}=1
$$

defines all scalar parameters. In the following derivation all parameters are restricted to be strictly positive. Let's express all unknowns in terms of $v_{\mathrm{f}}$. From simple algebra applied to Eqs. $(19,20)$ and then Eq. (21) we find

$$
D_{e}=\frac{x_{4}-D_{\mathrm{f}}^{2} v_{\mathrm{f}}}{x_{3}-D_{\mathrm{f}} v_{\mathrm{f}}}, v_{\mathrm{e}}=\frac{\left(x_{3}-D_{\mathrm{f}} v_{\mathrm{f}}\right)^{2}}{x_{4}-D_{\mathrm{f}}^{2} v_{\mathrm{f}}}, \Delta_{e}=\frac{x_{5}}{x_{3}-D_{\mathrm{f}} v_{\mathrm{f}}}
$$

The intra-axonal parameters are expressed from Eqs. $(17,18)$,

$$
D_{i}=\frac{x_{2}-\Delta_{e}^{2} v_{\mathrm{e}}}{x_{1}-\Delta_{e} v_{\mathrm{e}}}=\frac{x_{2} x_{4}-x_{5}^{2}-D_{\mathrm{f}}^{2} v_{\mathrm{f}} x_{2}}{x_{1} x_{4}-x_{3} x_{5}+D_{\mathrm{f}} v_{\mathrm{f}} x_{5}-D_{\mathrm{f}}^{2} v_{\mathrm{f}} x_{1}}
$$

and

$$
v_{\mathrm{i}}=\frac{\left(x_{1}-\Delta_{e} v_{\mathrm{e}}\right)^{2}}{x_{2}-\Delta_{e}^{2} v_{\mathrm{e}}}=\frac{\left(x_{1} x_{4}-x_{3} x_{5}+D_{\mathrm{f}} v_{\mathrm{f}} x_{5}-D_{\mathrm{f}}^{2} v_{\mathrm{f}} x_{1}\right)^{2}}{\left(x_{4}-D_{\mathrm{f}}^{2} v_{\mathrm{f}}\right)\left(x_{5}^{2}-x_{2} x_{4}+D_{\mathrm{f}}^{2} v_{\mathrm{f}} x_{2}\right)}
$$

Now, all expressions depend exclusively on the unknown $v_{\mathrm{f}}$. The last equation $v_{\mathrm{i}}+v_{\mathrm{e}}+v_{\mathrm{f}}=1$ solves for $v_{\mathrm{f}}$ as follows

$$
\begin{aligned}
1-v_{\mathrm{f}} & =v_{\mathrm{i}}+v_{\mathrm{e}} \\
& =\frac{D_{\mathrm{f}}^{2} v_{\mathrm{f}} x_{1}^{2}-x_{1}^{2} x_{4}-x_{2} x_{3}^{2}-D_{\mathrm{f}}^{2} v_{\mathrm{f}}^{2} x_{2}+2 x_{1} x_{3} x_{5}+2 D_{\mathrm{f}} v_{\mathrm{f}} x_{2} x_{3}-2 D_{\mathrm{f}} v_{\mathrm{f}} x_{1} x_{5}}{x_{5}^{2}-x_{2} x_{4}+D_{\mathrm{f}}^{2} v_{\mathrm{f}} x_{2}}
\end{aligned}
$$

Multiplying both sides by the denominator $\left(x_{5}^{2}-x_{2} x_{4}+D_{\mathrm{f}}^{2} v_{\mathrm{f}} x_{2}\right)$ (which is allowed since $x_{5}^{2}-x_{2} x_{4}+$ $\left.D_{\mathrm{f}}^{2} v_{\mathrm{f}} x_{2}=-D_{i}^{2} D_{e}^{2} v_{\mathrm{e}} v_{\mathrm{i}} \neq 0\right)$ leads to the following equation in $v_{\mathrm{f}}$

$$
\left(x_{2} D_{\mathrm{f}}^{2}-D_{\mathrm{f}}^{2} x_{1}^{2}+2 D_{\mathrm{f}} x_{1} x_{5}-2 x_{2} x_{3} D_{\mathrm{f}}-x_{5}^{2}+x_{2} x_{4}\right) v_{\mathrm{f}}+\left(x_{4} x_{1}^{2}-2 x_{1} x_{3} x_{5}+x_{2} x_{3}^{2}+x_{5}^{2}-x_{2} x_{4}\right)=0
$$

This equation is linear, since the quadratic terms in $v_{\mathrm{f}}$ cancel, which results in the unique final solution

$$
v_{\mathrm{f}}=\frac{x_{2} x_{4}-x_{2} x_{3}^{2}-x_{1}^{2} x_{4}-x_{5}^{2}+2 x_{1} x_{3} x_{5}}{x_{2} x_{4}+D_{\mathrm{f}}^{2} x_{2}-x_{5}^{2}-D_{\mathrm{f}}^{2} x_{1}^{2}-2 D_{\mathrm{f}} x_{2} x_{3}+2 D_{\mathrm{f}} x_{1} x_{5}}
$$

By inserting this $v_{\mathrm{f}}$ into Eqs. (23-25) we obtain the full solution for all parameters, which is our main result.

We now analyze the case of zero denominator in Eq. (29), which will express an ambiguity inherent to the model itself. Substituting the defining expression for the $x_{i}$ 's, Eqs. (17-21), gives for the denominator the form $v_{\mathrm{e}} v_{\mathrm{i}}\left(D_{i} D_{e}-D_{i} D_{\mathrm{f}}+\Delta_{e} D_{\mathrm{f}}\right)^{2}$ and the same form multiplied with $v_{\mathrm{f}}$ for the numerator. This means that for the special case

$$
D_{i} D_{e}-D_{i} D_{\mathrm{f}}+\Delta_{e} D_{\mathrm{f}}=0
$$

there is no information about $v_{\mathrm{f}}$, since Eq. (28) turns into an identity. In other words, the constraint $v_{\mathrm{i}}+v_{\mathrm{e}}+v_{\mathrm{f}}=1$ is automatically fulfilled for any $v_{\mathrm{f}}$. Note that the system of Eqs. (17-21) is linear 

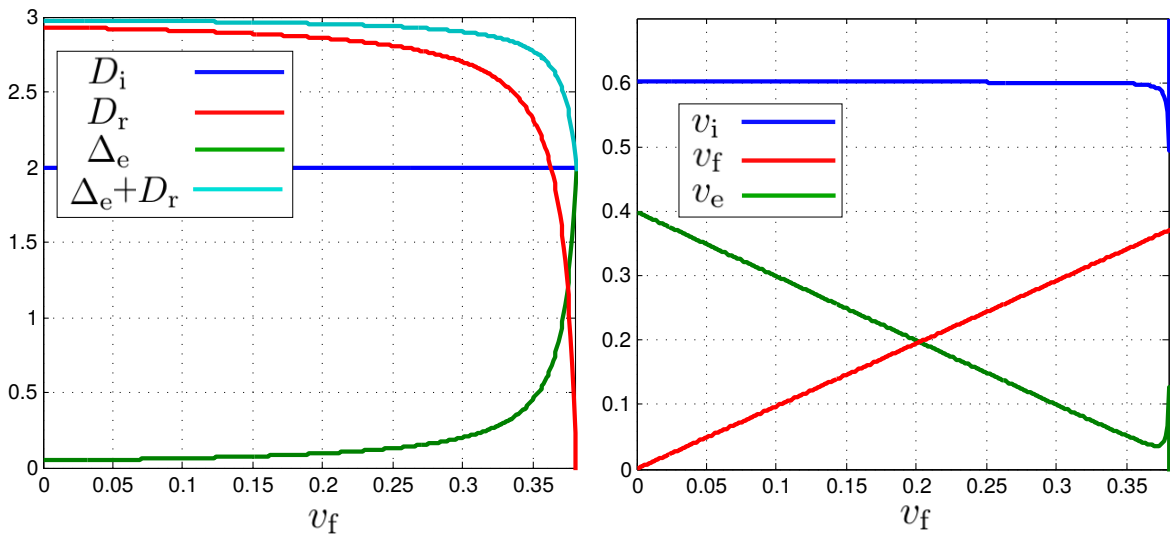

Figure 1: An example for a family of solutions where $D_{i} D_{e}-D_{i} D_{\mathrm{f}}+\Delta_{e} D_{\mathrm{f}}=0$ for varying $v_{\mathrm{f}}$. All these solutions have the same linear and planar moments up to order two.

in the volume fractions. In particular, given the diffusivities, Eqs. $(17,19,22)$ can be used to build a linear system for the volume fractions. The determinant of the so constructed system is just the left-hand side of Eq. (30) - its zero value implies a linear dependency, thus resulting in an infinite number of solutions.

To understand the physics behind the degeneracy condition, Eq. (30), consider first two special cases. If $D_{e}=0$, Eq. (30) gives $\Delta_{e}=D_{i}$, which means that the extra-axonal compartment is indistinguishable from the intra-axonal one. Another case is the isotropic extra-axonal compartment, $\Delta_{e}=0$, in which case it is indistinguishable from free water, $D_{e}=D_{\mathrm{f}}$. We found a family of solutions that interpolates between these two special cases, which is shown in Figure 1, as a functions of $v_{\mathrm{f}}$. This solution only exists for a specific choice of diffusivities obeying Eq. (30). All the shown solutions have exactly the same moments up to second order. Outside the displayed interval, the solution is unphysical with several negative parameters. Interestingly, the intra-axonal diffusivity is not subjected to the ambiguity. In that case, one can find $D_{i}=\frac{D_{\mathrm{f}} x_{2}}{D_{\mathrm{f}}-x_{5}}$.

Note the similarity of the above degeneracy to the bi-exponential model when the diffusivities in two compartments are equal. Equation (30) expresses this inherent drawback of multi-exponential models exemplified by the standard white matter model.

\subsection{Determination of mesoscopic dispersion $f_{2}$}

Equation (16) operates with the moments of the order $b^{2}$. Here we show that the dispersion can also be expressed directly in terms of the signal. Recall that the moments $W_{\alpha}^{l, k}$ define the Taylor expansion of $S_{\alpha}^{l}(b)$ in powers of $b$ according to Eq. (8). Therefore the function

$$
F(b):=-\frac{7}{4} \frac{S_{\mathrm{lin}}^{2}(b)-2 S_{\mathrm{pla}}^{2}(b)}{S_{\mathrm{lin}}^{0}(b)-S_{\mathrm{pla}}^{0}(b)}
$$

reproduces Eq. (16) with account for the identities $W_{\text {lin }}^{0,1}=W_{\text {pla }}^{0,1}$ and $W_{\text {lin }}^{2,1}=2 W_{\text {pla }}^{2,1}$. Practically, one has to consider the function

$$
F(b)=f_{2}+\mathcal{O}(b)
$$

and fit it linearly to find its value for $b=0$.

\subsection{Linear and spherical encodings are not sufficient}

For spherical encoding we have

$$
\begin{aligned}
S_{\mathrm{sph}}(b) & =v_{\mathrm{i}} e^{-b D_{i} / 3}+v_{\mathrm{e}} e^{-b\left(\Delta_{e} / 3+D_{e}\right)}+v_{\mathrm{f}} e^{-D_{\mathrm{f}} b} \\
W_{\mathrm{sph}}^{k} & =\left.\frac{d^{k}}{d b^{k}}\right|_{b=0} S_{\mathrm{sph}}(b)
\end{aligned}
$$



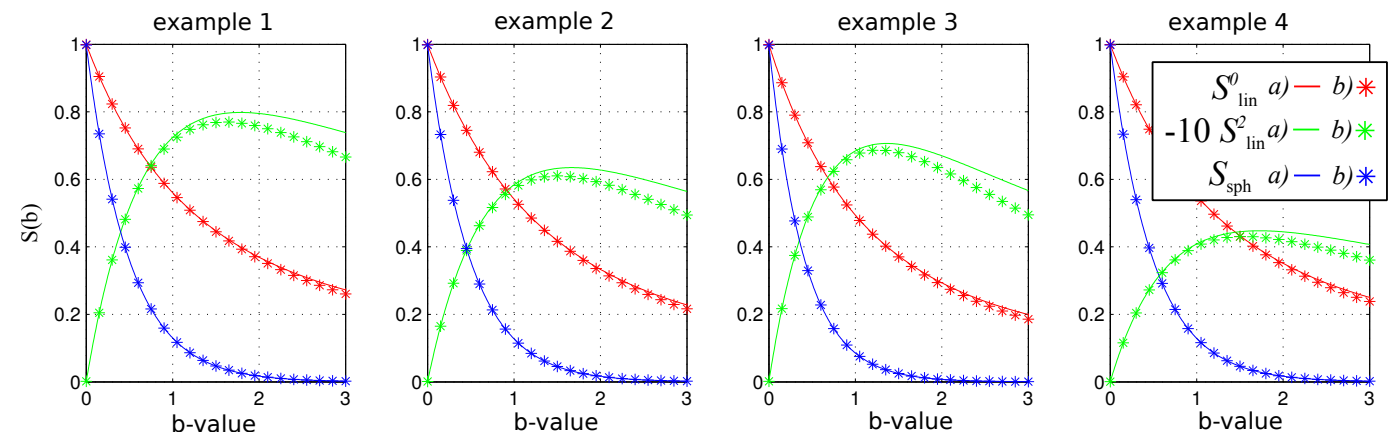

Figure 2: Signal courses for the four examples, where linear and spherical moments are identical up to order two. First notable differences appear above $b=2$. Note that for $S_{\text {lin }}^{2}$ differences are enlarged by a factor of ten.

We assume that only moments up to $\mathcal{O}\left(b^{2}\right)$ are observable, i.e. $W_{\text {lin }}^{0,1}, W_{\text {lin }}^{0,2}, W_{\text {lin }}^{2,1}, W_{\text {lin }}^{2,2}, W_{\mathrm{sph}}^{1}, W_{\mathrm{sph}}^{2}$ are known. We know that $W_{\mathrm{sph}}^{1}$ is linearly dependent on $W_{\text {lin }}^{0,1}$ and $W_{\text {lin }}^{0,2}$, so linear and spherical encodings give five equations up to order 2. In fact, with these equations, one can find analytically a solution for the two-compartment model without the fast water fraction. However, this solution has two roots and is, hence, ambigious. We do not show here the solutions, but give a few numeric examples, where both roots lead to physical meaningful results:

\begin{tabular}{l|l|l|l|l|l} 
solution & $D_{i}$ & $\Delta_{e}$ & $D_{e}$ & $f_{2}$ & $v_{\mathrm{i}}$ \\
\hline $1 . \mathrm{a}$ & 2.00 & 0.60 & 0.50 & 0.80 & 0.60 \\
$1 . \mathrm{b}$ & 2.11 & 1.29 & 0.24 & 0.74 & 0.31 \\
\hline $2 . \mathrm{a}$ & 2.00 & 0.60 & 0.50 & 0.80 & 0.40 \\
$2 . \mathrm{b}$ & 2.17 & 1.11 & 0.31 & 0.72 & 0.17 \\
\hline $3 . \mathrm{a}$ & 2.40 & 1.00 & 0.50 & 0.80 & 0.40 \\
$3 . \mathrm{b}$ & 2.58 & 1.51 & 0.31 & 0.75 & 0.14 \\
\hline $4 . \mathrm{a}$ & 2.00 & 0.60 & 0.50 & 0.50 & 0.50 \\
$4 . \mathrm{b}$ & 2.14 & 1.20 & 0.27 & 0.46 & 0.24 \\
\hline
\end{tabular}

where mainly $v_{\mathrm{i}}, \Delta_{e}$ and $D_{e}$ are confused. The parameters $D_{i}, f_{2}$ and $\Delta_{e}+D_{e}$ are rather stable. This goes in line with the observation that a spherical encoding can resolve the ambiguity of the parallel diffusivities [Fieremans et al., 2011, Fieremans et al., 2018, Dhital et al., 2017], but still has to struggle with $\Delta_{e}, D_{e}$ and $v_{\mathrm{i}}$. In Figure 2 we show signal courses for the counterexamples.

\section{Conclusion}

We have constructively shown that linear and planar diffusion encodings can fully resolve the three-compartment model of white matter using data up to the order $\mathcal{O}\left(b^{2}\right)$ and $l=2$. The common experience with the diffusional kurtosis imaging [Jensen et al., 2005] indicates the practical availability of $\mathcal{O}\left(b^{2}\right)$ terms. While in principle, these terms include information for $l \leq 4$, the order $l=4$ is spoiled by noise as it was shown for a typical two-shell measurement on an advanced scanner with the maximal gradient strength $80 \mathrm{mT} / \mathrm{m}$ [Reisert et al., 2017, Fig. 2].

Our analysis highlighted a special situation of ambiguous solution due to an inherent inability of multiexponential models to resolve compartments with similar parameters. The only way to distinguish such compartments is measuring in a domain where their differences get apparent, for example in the large b-value regime, where stable estimates of higher order information becomes possible. Without such information, a stable parameter estimate is only possible relying on prior knowledge.

We have also shown that a combination of spherical and linear encoding is not enough to find a unique solution in order $\mathcal{O}\left(b^{2}\right)$. In fact, $\mathcal{O}\left(b^{2}\right)$ information delivered by a spherical encoding is fully contained in the combination of linear and planar information, namely $W_{\mathrm{sph}}^{0,2}=\left(4 W_{\mathrm{pla}}^{0,2}-W_{\text {lin }}^{0,2}\right) / 3$, which renders a spherical encoding in the presence of linear and planar encodings in the low bvalue regime superfluous. In fact, it is a fortunate coincidence that $\mathcal{O}\left(b^{2}\right)$ information spanned by 
linear and planar diffusion encoding (it is actually the 'full' encoding in $\mathcal{O}\left(b^{2}\right)$ ) is 6 dimensional (Eqs. (10-15)) and the parameter space of the three compartment white matter model has also 6 free parameters, Eq. (2).

The derived mapping is only valid for noiseless signals, i.e., when the signal is in the image of the modeling equation. For practical applications the obtainable signal-to-noise ratios are too low. A recent preprint [Coelho et al., 2018] shows by numerical simulations that in a slightly simplified setting (two-compartments and Watson distribution) also in the noisy case the degeneracy is resolved. The importance of the analytical solution lies in its justification for parameter estimators that rely on unimodal posterior distributions. Additionally, the solution can give certain hints for the construction of such parameter estimators. In fact, the expression of the parameters are all low-order rational functions of the moments (which are all linear projections of the signal). This suggests to make a similar approach for the estimator (e.g. as found in [Reisert et al., 2017]), i.e. using functions, which are rational in linear combinations of the signal.

\section{References}

[Coelho et al., 2017] Coelho, S., Beltrachini, L., Pozo, J., and Frangi, A. (2017). Double diffusion encoding vs single diffusion encoding in parameter estimation of biophysical models in diffusionweighted mri. In Proceedings of the ISMRM, Honolulu.

[Coelho et al., 2018] Coelho, S., Pozo, J. M., Jespersen, S. N., Jones, D. K., and Frangi, A. F. (2018). Double diffusion encoding prevents degeneracy in parameter estimation of biophysical models in diffusion mri. arXiv preprint arXiv:1809.05059, submitted to MRM.

[Dhital et al., 2017] Dhital, B., Kellner, E., Kiselev, V. G., and Reisert, M. (2017). The absence of restricted water pool in brain white matter. Neuroimage.

[Dhital et al., 2018] Dhital, B., Reisert, M., Kellner, E., and Kiselev, V. G. (2018). Diffusion weighting with linear and planar encoding solves degeneracy in parameter estimation. In Proceedings of the ISMRM, Paris.

[Fieremans et al., 2011] Fieremans, E., Jensen, J. H., and Helpern, J. A. (2011). White matter characterization with diffusional kurtosis imaging. Neuroimage, 58(1):177-188.

[Fieremans et al., 2018] Fieremans, E., Veraart, J., Benjamin, A.-A., Filip, S., Nilsson, M., and Novikov, D. (2018). Effect of combining linear with spherical tensor encoding on estimating brain microstructural parameters. In Proceedings of the ISMRM, Paris.

[Jelescu et al., 2016] Jelescu, I. O., Veraart, J., Fieremans, E., and Novikov, D. S. (2016). Degeneracy in model parameter estimation for multi-compartmental diffusion in neuronal tissue. NMR Biomed, 29(1):33-47.

[Jensen et al., 2005] Jensen, J. H., Helpern, J. A., Ramani, A., Lu, H., and Kaczynski, K. (2005). Diffusional kurtosis imaging: The quantification of non-gaussian water diffusion by means of magnetic resonance imaging. Magn. Reson. Med., 53:1432-1440.

[Jespersen et al., 2013] Jespersen, S. N., Lundell, H., Sønderby, C. K., and Dyrby, T. B. (2013). Orientationally invariant metrics of apparent compartment eccentricity from double pulsed field gradient diffusion experiments. NMR in Biomedicine, 26(12):1647-1662.

[Lampinen et al., 2017] Lampinen, B., Szczepankiewicz, F., Mårtensson, J., van Westen, D., Sundgren, P. C., and Nilsson, M. (2017). Neurite density imaging versus imaging of microscopic anisotropy in diffusion mri: a model comparison using spherical tensor encoding. Neuroimage, 147:517-531.

[Novikov et al., 2018] Novikov, D. S., Veraart, J., Jelescu, I. O., and Fieremans, E. (2018). Rotationally-invariant mapping of scalar and orientational metrics of neuronal microstructure with diffusion mri. NeuroImage, 174:518-538.

[Reisert et al., 2017] Reisert, M., Kellner, E., Dhital, B., Hennig, J., and Kiselev, V. G. (2017). Disentangling micro from mesostructure by diffusion mri: A bayesian approach. Neuroimage, 147:964-975. 
[Reisert et al., 2018] Reisert, M., Kiselev, V. G., and Dhital, B. (2018). Unconstrained estimation of microstructure by the combination of single- and double-planar diffusion encoding. In Proceedings of the ISMRM, Paris.

[Szczepankiewicz et al., 2015] Szczepankiewicz, F., Lasič, S., van Westen, D., Sundgren, P. C., Englund, E., Westin, C.-F., Ståhlberg, F., Lätt, J., Topgaard, D., and Nilsson, M. (2015). Quantification of microscopic diffusion anisotropy disentangles effects of orientation dispersion from microstructure: applications in healthy volunteers and in brain tumors. NeuroImage, 104:241-252.

[Westin et al., 2014] Westin, C.-F., Szczepankiewicz, F., Pasternak, O., Özarslan, E., Topgaard, D., Knutsson, H., and Nilsson, M. (2014). Measurement tensors in diffusion mri: generalizing the concept of diffusion encoding. In International Conference on Medical Image Computing and Computer-Assisted Intervention, pages 209-216. Springer.

[Zhang et al., 2012] Zhang, H., Schneider, T., Wheeler-Kingshott, C. A., and Alexander, D. C. (2012). Noddi: practical in vivo neurite orientation dispersion and density imaging of the human brain. Neuroimage, 61(4):1000-1016. 